\title{
Akademische oder nichtakademische Psychotherapie?
}

\author{
Michael Linden \\ Rehabilitationszentrum Seehof der Deutschen Rentenversicherung Bund, Teltow/Berlin, Deutschland
}

In diesem Heft findet sich ein Interview mit Prof. Lenz aus Wien, in dem er die wesentlichen Eckpunkte der Psychotherapie-Ausbildung und -Anwendung in Österreich kurz darstellt. In Österreich können auch Sozialarbeiter und Lehrer und nach Einzelfallprüfung auch jeder andere Psychotherapeut werden. Bei genauerer Betrachtung ist die Situation in Deutschland nicht sehr viel anders. Neben Ärzten und Psychologischen Psychotherapeuten erbringen auch Sozialarbeiter, beispielsweise in der Suchttherapie oder in Beratungsstellen, oder Pädagogen und Ergotherapeuten in der Kindertherapie psychotherapeutische Leistungen. Schließlich ermöglicht das Heilpraktikergesetz letztlich ebenfalls jedermann, sich als «Psychotherapeut» zu bezeichnen und Krankenbehandlungen durchzuführen und dies teilweise auch mit Bezahlung durch Krankenkassen. Dabei werden nicht einmal spezifische Ausbildungsanforderungen verlangt. Auch in anderen Ländern, z.B. den Beneluxstaaten, Großbritannien, Skandinavien oder den USA werden vielfältige Varianten von Psychotherapie durch unterschiedlichste nichtakademische Berufsgruppen erbracht. Schließlich findet man in Deutschland in Kliniken vielfältige Beispiele, dass eindeutig psychotherapeutische Leistungen, oft aus Kostengründen, auf andere Berufsgruppen übertragen werden.

Daher stellt sich mit Blick auf die Wirtschaftlichkeit, die Versorgungsrealität, das Indikationsspektrum und sozialpsychologische und wissenschaftliche Überlegungen die Frage, warum die Erbringung von Psychotherapie nicht grundsätzlich für jedermann geöffnet werden sollte. Unter wirtschaftlichen Gesichtspunkten wären psychotherapeutische Leistungen durch Nicht-Akademiker deutlich günstiger. Statt für 80 Euro pro Sitzung könnte man schon für 20 Euro Zuwendung und Unterstützung erhalten, d.h. für das gleiche Geld könnten viermal so viele Menschen Hilfe bekommen. Des Weiteren zeigt die Versorgungsrealität in Deutschland wie andernorts, dass außer Ärzten und Psychologischen Psychotherapeuten offenbar auch andere Berufsgruppen kompetent Psychotherapie erbringen können. Mit Blick auf das Indikationsspektrum ist anzumerken, dass trotz des Gutachterverfahrens ein sehr breites Spektrum an Störungen zur Behandlung kommt, einschließlich allgemeiner Lebensprobleme. Sozialpsychologisch ist noch darauf hinzuweisen, dass ein Großteil der Patienten keine Akademiker und erst recht keine Psychologen oder Ärzte sind, sodass Therapeuten mit anderen Grundberufen möglicherweise sogar die besseren Ratgeber sein könnten, weil sie näher an der Lebenswelt ihrer Patienten sind. Schließlich fehlen auch wissenschaftlich bislang Belege, dass eine akademische Psychotherapie relevant bessere Ergebnisse erzielt als eine nichtakademische Psychotherapie.

Was spricht also für Ärzte und Diplompsychologen bzw. akademische Psychotherapeuten? Für Ärzte gilt zunächst einmal ein versorgungstechnisches Argument: Zu einer ganzheitlichen, umfassenden und mehrdimensionalen Krankenbehandlung gehören neben somatomedizinischen, pharmakotherapeutischen und sozialmedizinischen Maßnahmen unverzichtbar auch psychotherapeutische Interventionen. Des Weiteren sind psychische Störungen Erkrankungen, die nur eingebettet in eine umfassende Krankheitslehre differenzialdiagnostisch adäquat verstanden und behandelt werden können. Für Psychologie als Grundberuf für Psychotherapeuten wird als wesentliches Argument vorgetragen, dass Psychologie die Wissenschaft ist, die die menschliche Psyche erforscht und menschliches Verhalten beschreibt. Die Fachgruppe Klinische Psychologie und Psychotherapie der Deutschen Gesellschaft für Psychologie fordert in einer Stellungnahme vom 24.02.2010, dass ein «Masterniveau als Eingangsvoraussetzung für den Zugang zur Ausbildung in Psychotherapie» unverzichtbar sei, um über «die Fähigkeit zu wissenschaftlich fundierten Entscheidungen im Bereich Diagnostik und Therapie» zu verfügen; ein Bachelor-Abschluss genügt demnach nicht. Ohne profunde Kenntnisse in Grundlagen der Wahrnehmung, des Denkens, der Emotion und Motivation, Entwicklungspsychologie, Differenzieller und Persönlichkeitspsychologie, Sozialpsychologie, Methodik und Anwendung der Diagnostik, Methodenlehre, Versuchsplanung, Epidemiologie, Evaluation und Pädagogische Psychologie würde der Beruf des Psychotherapeuten insgesamt «deutlich an akade-

\section{KARGER}

Fax +497614520714

Information@Karger.de

www.karger.com
(C) 2010 S. Karger GmbH, Freiburg

Accessible online at:

www.karger.com/ver
Prof. Dr. Michael Linden

Rehabilitationszentrum Seehof der Dt. Rentenversicherung Bund Lichterfelder Allee 55

14513 Teltow/Berlin, Deutschland

Tel. +493328345678 , Fax 345555

michael.linden@zedat fu-berlin.de 
mischer Qualifikation verlieren und es würde der bisherige Standard der psychologischen Psychotherapie verlassen werden» [Deutsche Gesellschaft für Psychotherapie, 2010].

Die Frage, wie aufwändig eine Psychotherapeutenausbildung sein muss, ist von großer gesellschaftlicher und gesundheitspolitischer Bedeutung und bislang eher durch Hinweis auf Traditionen, denn durch Fakten zu beantworten. Die Frage stellt sich zudem für das gesamte Gesundheitswesen, wo auch für Ärzte zunehmend nach einfacher zu gewinnenden Therapeuten gesucht wird, z.B. Gemeindeschwestern als Arztersatz in ländlichen Regionen oder Anästhesiepfleger, die weitgehend eigenständig Narkosen durchführen.

Was folgt daraus? Ohne Anspruch auf Vollständigkeit lassen sich drei Punkte hervorheben:

1. Es ist dringend erforderlich, eine empirische Ausbildungsforschung zu betreiben. Die psychotherapeutischen Wissenschaften bringen dafür alle fachlichen Voraussetzungen mit.

2. Hohe Ausbildungsvoraussetzungen für Psychotherapie sind nur zu rechtfertigen, wenn Psychotherapie sehr viel konsequenter auf Krankenbehandlung im engeren Sinne eingeschränkt wird, und supportive Therapie oder die Unterstützung bei der Bewältigung allgemeiner Lebensbelastungen anderen überlassen wird.

3. Es muss gefordert werden, dass Psychotherapeuten deutlich anderes tun als wohlmeinende Helfer. Eine gute Therapeut-Patient-Beziehung alleine, wie sie von integrativen Psychotherapiemodellen als zentrales Heilmittel propagiert wird, wird nicht hinreichen können.

\section{Literatur}

Deutsche Gesellschaft für Psychologie (DGPS), Fachgruppe Klinische Psychologie und Psychotherapie: Stellungnahme vom 24.02.2010. 